\title{
Synthesis and Characterization of Cement/Carbon-Nanotube Composite for Structural Health Monitoring Applications
}

\author{
Diego Castaneda-Saldarriaga ${ }^{1}$, Julián Sierra-Pérez' ${ }^{1}$, Joham Alvarez-Montoya ${ }^{1}$ \\ ${ }^{1}$ Grupo de Investigación en Ingeniería Aeroespacial, Universidad Pontificia Bolivariana \\ Sede Medellín, Circular 1a 70-01, Medellín, Colombia \\ diego.castanedas@upb.edu.co; julian.sierra@upb.edu.co; joham.alvarez@upb.edu.co
}

\begin{abstract}
The civil structures during their useful life are subjected to different loads (environmental and mechanical), that cause progressively deterioration and that consequently requires maintenance efforts to keep them safe and operative. In this work, a novel alternative is proposed based in the development of a smart material with multifunctional characteristics that allow the Structural Health Monitoring (SHM)without requiring external sensors. This was possible by developing cement matrix compounds with the addition of carbon nanotubes. The manufacturing of this material began with an experimental design (DOE) that allowed to determine the mixturestype with which the sensor was manufactured, additionally repeatability and reproducibility was guaranteed. To measure the piezoresistive behavior of the samples, we used an INSTRON 5582 universal testing machine, a data acquisition equipment to obtain the variation in voltage and Micron Optics SM130 optical sensing interrogator for measuring deformations. Once the rheological and mechanical behavior of the material were characterized, mechanical tests of the material were carried out without exceeding the maximum deformations of the material in order to acquire data on the resistive behavior of the structure and find the gauge factor. The results of this development offer a part of the solution to the growing needs in the field of civil engineering against the early warning of damages caused by both natural phenomena and human causes (use of inappropriate materials, wrong calculations, overloads).
\end{abstract}

Keywords: Sensor development, Smart material, SHM, DOE, Piezoresistive, Gauge Factor.

\section{Introduction}

The demographic increase has generated rapid vertical growth in different cities around the world due to the limited surface availability in contrast to its high demand for construction-related uses [1]. In addition, new structural challenges such as loads induced by movements or reactivation of geological faults induced by the civil construction activity have begun to be reported [2].

Indeed, buildings are under constant loading stress (operational and environmental loads), which can generate shortterm damages and long-term implications on the performance of the civil structure [3]. The identification of these damages can be difficult because they appear anywhere at any time and detecting them timely can be complicated, so it is common that at the moment of identifying them, they already represent a risk for the operation of the infrastructure.

Currently, methods based on non-destructive testing (NDT) techniques are mostly used to assess the structural integrity of engineering components. However, these techniques are costly to implement due to the high operating costs associated with the equipment, they are high time consuming to collecting information and many of them depend on human supervision for decision making, decreasing the degree of reliability and increasing the uncertainty in the results obtained [4]. Therefore, some NDT-based methodologies become inefficient when automatic structural health monitoring (SHM) methodologies are required.

Structural health monitoring techniques seek to provide information on a structure's integrity, minimizing the need for programmed inspections, allowing in-situ measure and providing continues structural state information [5].

SHM can be defined as a set of fault evaluation techniques, which consist on the identification of four characteristics that are related to the physical state of an engineering component while it operates and without the identification affectation of the structure's integrity [6]-[8]. These are:

- The operational and environmental loads that interact with the component or system,

- The damages caused by these loads,

- The growth of the damage as the component or system operates and 
- The performance of the component or system as damage accumulates during service.

One of the main challenges faced by the SHM techniques in civil engineering field is the research and development of robust sensors that allow the four previous characteristics to be measured over time using periodically spaced dynamic response and instrumented in a non-invasive way inside the engineering component [6].

One solution to this problem is the development of a smart material (for example a composite material) capable of reacting to external stimuli such as external stress, geometry changes and environment variables. The capability to include different types of fillers in metal, ceramic or polymer matrices has led to generate novel solution to the problem, ranging from heating elements, structural reinforcement to sensor development [5], [9]-[11].

In this context, Carbon Nanotubes (CNTs) inclusion inside ceramic matrix, such as cement, can generate a selfsensing composite, using as a principle of operation the variation in electrical resistance when external loads are applied to its structure, known as piezoresistive effect [12]-[16]. CNTs cement-based sensors have been proposed as an option to obtain a mechanically strong bond with the monitored structure, obtaining information from the civil engineering structure and creating a continuously distributed set of strain sensors within the structure[17], [18].

The construction of cement matrix sensors with inclusions of CNTs requires control of several variables, such as the dispersion of the CNTs, the hydration time of the concrete, the plasticity of the concrete and the particle sizes of the aggregates. From this, several studies have been published around the characterization of the piezoresistive effect from multiples cement/CNTs composites samples, varying the water/cement (w/c) ratio, the surfactant proportion to avoid the agglomeration effect and the CNTs proportion between the composite to obtain a decent gauge factor response [17], [19], [20].

This paper is aimed at presenting a multi-purpose study dealing with the experimental behavior characterization of cement with different surfactant's types, taking into account that the control variable was the maximum stress obtained and the fluidity of it (i), setting parameters for a four points probe methods in order to find the electrical resistance behavior against the variation of CNT volume fraction inside the composite (ii), and piezoresistive strain sensing characterization, in order to provide information around the potential applications (iii). To this aim, the present work presents materials and methods (Section 2), the results and discussions considering the multiple characterizations (Section 3), and the key conclusions are reported in the final Section 4.

\section{Materials and Methods}

In this work, two types of concrete were manufactured. Concrete Type 1 was manufactured without CNT load, in order to find an optimal relationship between the workability of the concrete and the use of surfactants. Concrete Type 2 was manufactured using carbon nanotubes, to generate piezoresistivity effect on the samples.

The cementitious material used in this study was ordinary Portland cement and as aggregate for the concrete, river quartz sand was used. Purified multiwall carbon nanotubes (MWCNTs) were used as reinforcement conductive filler. The length of the MWCNT was in the range of 10-30 $\mu \mathrm{m}$. As surfactants was used SDS, Tween 20 and triton X-100 were used, which proved highly effective for CNT dispersion and rendered employment of conventional dispersion methods. Surfactant are commercially available by PanReac AppliChem.

Prismatic specimens, with dimensions of $52 \times 52 \times 52 \mathrm{~mm}^{3}$ were fabricated following the ASTM C109/C109M: 2016a for each mixture/loading value. The materials used in the manufacturing process shown in Fig. 1 are listed in Table 1. The aging of the concrete was made taking into account the standard 308R-16.

\subsection{Cement workability}

According to [21] and [20], to achieve CNTs dispersion it is necessary a workability control of the cement/water/CNTs mixture. Therefore, this parameter will be assumed as critical and will be assigned priority over parameters associated with the mechanical behavior of the sample. To achieve the first parameters, a design of experiment (DOE) was applied using as the input variable the type of surfactant and the water/surfactant ratio (w/d), the w/d ratio was set as 5\%,10\% and 15\% and two repetitions were carried out.

These three plasticizers have been reported in different studies as good dispersing agents of carbon nanotubes, therefore any election that results from the DOE, will produce a positive effect on the dispersion of the CNTs within the cement matrix. 
The DOE results previously proposed, is the generation of 27 concrete samples, in order to apply two tests, workability test and compression test. The workability is a property of fresh concrete that refers to the ease with which this can be mixed, handled, transported, among others, without losing its homogeneity [22].

Table 1: Materials and quantities.

\begin{tabular}{|c|c|c|}
\hline Material & Specification & $\begin{array}{c}\text { Proportion for the } \\
\text { manufacture of } 1 \text { cube }\end{array}$ \\
\hline Cement & Portland type & $83 \mathrm{~g}$ \\
\hline sand & Quartz sand & $229 \mathrm{~g}$ \\
\hline Water & Ordinary water & $40.3 \mathrm{~g}$ \\
\hline \multirow{3}{*}{ Surfactant } & Tween 20 & \multirow{3}{*}{$\begin{array}{c}\text { the weight of the } \\
\text { surfactant depends } \\
\text { directly on the } \\
\text { water/surfactant ratio. }\end{array}$} \\
\hline & sodium dodecyl sulfate (SDS) & \\
\hline & Triton $\mathrm{X}-100$ & \\
\hline
\end{tabular}

In order to prepare a cementitious material that is as much homogeneous as possible, the fabricating process was divided into two steps. Firstly, a solution of water and surfactant was created (Fig. 1(a)), then, the cement is continuously added to the water/surfactant suspension at a rate of $83 \mathrm{~g} / \mathrm{min}$ while mechanical mixing is applied at a rate of $100 \mathrm{rev} / \mathrm{min}$. (Fig. 1(c) and $1(\mathrm{~d}))$. Secondly, the concrete mixing is poured into a $10 \mathrm{~cm}$ cylinder height and $5.08 \mathrm{~cm}$ wide cylinder and is compressed according to the ASTM C109/C109M: 2016a norm (Fig. 1(e)). Next, the cylinder is removed and the content of this (concrete mix) collapses on itself. At this moment a frontal photograph is taken to later be processed and obtain the dispersed area of concrete (Fig. 1(f)), poured into a mold (Fig. 1(g)). After solidification, the samples were unmolded for curing in laboratorycontrolled conditions. Fig. 1 illustrates the step-by-step concrete composite fabricating process and concrete fabricating for fluency test.

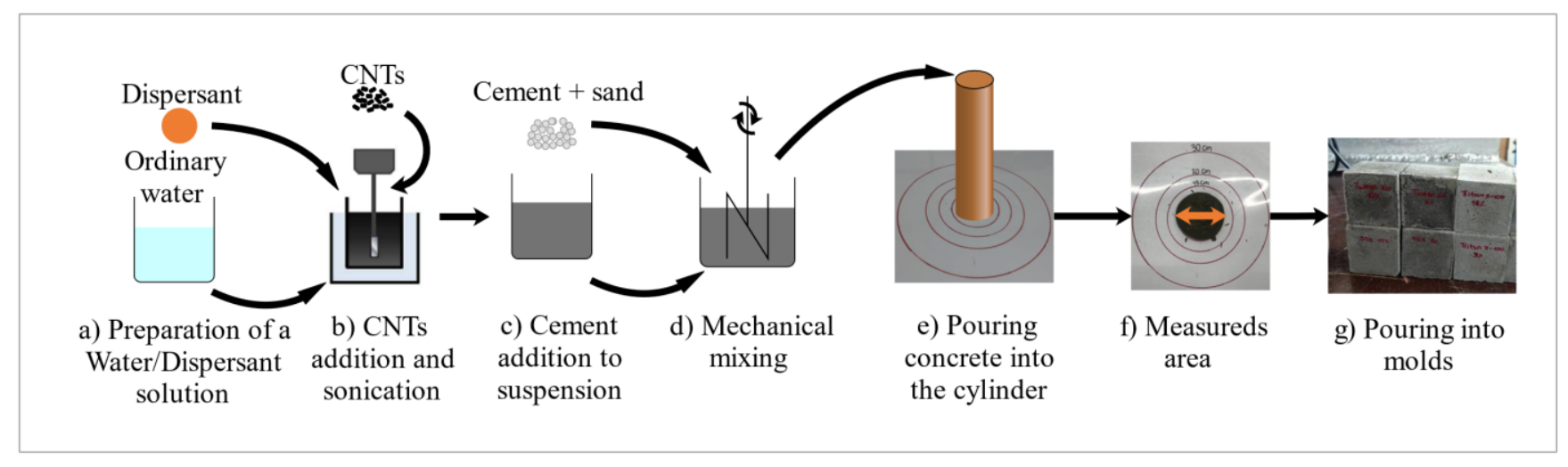

Fig. 1: Concrete fabricating process for concrete fluency test.

\subsection{Carbon Nanotubes/Water suspension preparation}

To improve CNTs dispersion into aqueous matrix and avoid CNTs agglomeration due to Van der Waals forces, suspensions are prepared by mixing the CNTs in deionized aqueous solution containing the surfactant and the resulting dispersions are sonicated at room temperature [23]. Energy is applied to the samples by a 250W high-intensity ultrasonic processor with a cylindrical tip. Sonicator is operated at $15 \%$ of the amplitude in order to deliver $12 \mathrm{~J}$ of energy, for a time of $30 \mathrm{~min}$, with 1 second on and two seconds off period in order to prevent overheating of the suspensions.

\subsection{Electric resistance response characterization.}

During the composite manufacturing with CNTs charge, copper anodes were embedded in order to collect information associated with the electrical behavior. Electric resistance characterization methodology setup was made on the following 
work [24]. The samples electric resistance was measured by connecting an external DAQ system to electrodes embedded in the cement/CNTs composite. To find the electric resistance, the samples were connected to a power source providing square wave potential pulses across their electrodes, with a $10 \mathrm{~V}$ as input voltage and 1 and $10 \mathrm{~Hz}$ as frequency.

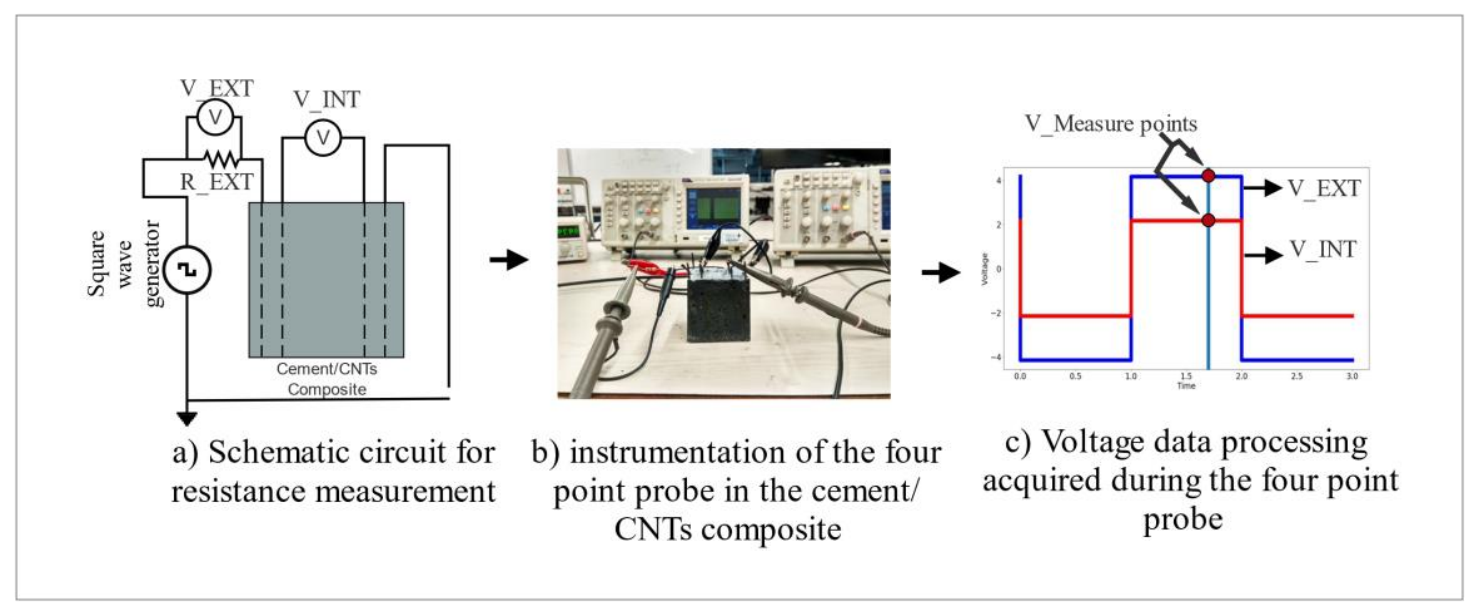

Fig. 2: Experimental setup for acquisition of electrical resistance of the samples.

The input voltage crosses through a resistance of known value (R_EXT $=2,2 \mathrm{k} \Omega, 100 \Omega$ and $10 \Omega$ ) and a digital voltmeter registers the power drop (V_EXT) value with which, applying the Ohm's equation, it is possible to find the current that flows the cement matrix composite. A second digital voltmeter is connected between of the center electrodes, with which the voltage that crosses the sample was measured, once this value is found, the Ohm's law is used to find the sample resistance.

The DAQ system used provides 50 data points measured by positive and negative pulse when the input frequency is $1 \mathrm{~Hz}$ and 10 when $10 \mathrm{~Hz}$ is used. The next methodological step was to select a percentage of the data measured in the positive pulse cycle, with which the voltage related to that percentage was extracted and Ohm's law is applied to find the current along the sample, as well as the resistance of the sample (Fig. 2(c)). Fig. 2 illustrates the step-by-step electric resistance measure process (Fig. 2(a) and 2(b)) and processing data step (Fig. 2(c)).

The electrical resistance was measured for the samples of $0.2 \%, 0.5 \%$ and $0.8 \%$ CNTs ratio, using 3 external resistance types and two sampling frequencies types, obtaining 18 experiment configurations set.

\subsection{Piezoresistive sample characterization}

In order to assess the mechanical and piezoresistive behavior of cement/CNTs composite, an axial strength measurement was applied using a universal testing machine type INSTRON 5582 (INSTRON, United Kingdom). Fig. 3 show the experimental setup for validation of the Material's strain-sensing capability.

For the piezoresistive test, an optical fiber sensor was bonded in the composite along the axis where the load was applied in order to measure the strain $\varepsilon$. The optical fiber sensor used was fiber Bragg gratings (FBG), which allow to measure strain by tracking the shifted reflected wavelength. Then, the strains acquired using a Micron Optics SM130 optical sensing interrogator were related to the variations in the resistivity measurements, then, using the Eqs. (1) it is possible to find the gauge factor of the material.

$$
\lambda=\frac{\Delta R}{\varepsilon R}
$$

Where $R$ the measured resistance without applied load, $\Delta R$ is the incremental variation in electrical resistance caused by the axial strain, $\lambda$ is the material's gauge factor and $\varepsilon$ is the strain. 


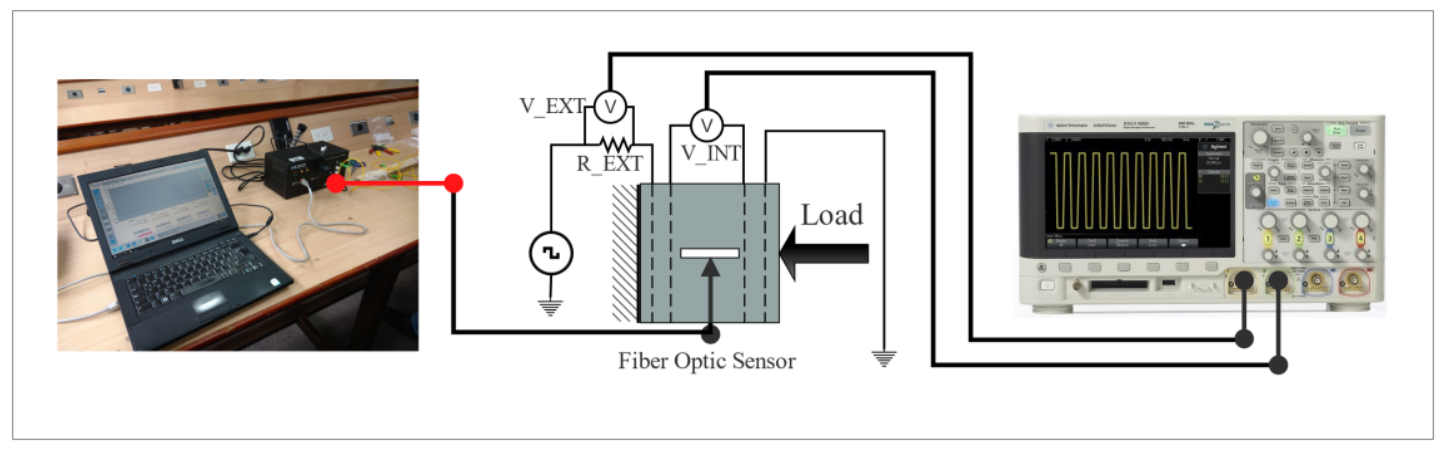

Fig. 3: Experimental setup for piezoresistivity measurement.

\section{Results and discussion}

\subsection{Mechanical and fluidity measurements}

Fig. 4(a) shows the mechanical response of the concrete samples with SDS, Triton and Tween surfactants when a compressive load is applied uniaxially. In particular, samples with surfactant type Tween resist a greater stress to compression, followed by the samples with surfactant type Triton and finally SDS. It can also be observed that the ratio between surfactant proportion and maximum effort is inversely proportional.
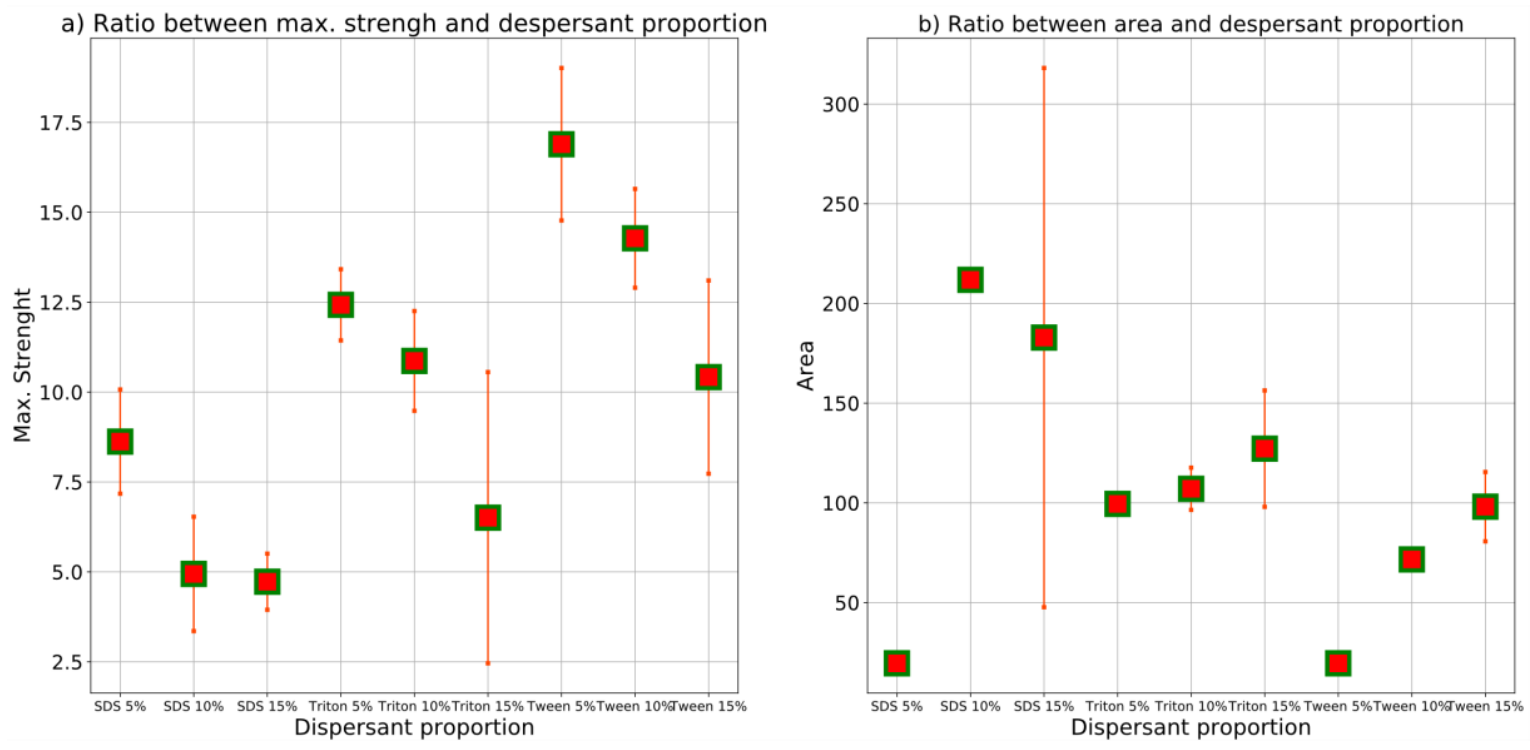

Fig. 4: DOE result for manufactures samples.

The decrease in mechanical resistance as the proportion of surfactant increases show in Fig. 4(a) is a consequence of using proportions above the critical radio of surfactant [25], however, priority was given to obtain a mixture with a high workability against mechanical properties of the sample.

On the other hand, the relationship between the dispersed area (fluidity) with the type of surfactant and its percentage showed in Fig. 4(b), demonstrate a directly proportional relationship for all the experiments. It was found that samples with SDS surfactant have the best workability. Consequently, the SDS at $10 \%$ volume fraction was selected to manufacture the concrete samples with CNT inclusions. This behavior is attributed to the solubility limit of the concrete solid suspension as opposed to the dispersant or critical volume, which when exceeded generates a decrease in the mechanical properties of the material [25]. 


\subsection{Electrical resistance measurements.}

In Fig. 5 is presented the electrical resistance characterization is presented when $V_{-} E X T$ and $V_{-} I N T$ are taken from a 0.2 to 0.9 range of the positive cycle in the input signal. It can be observed that the standard deviation is greater if the endpoints of the evaluated range are used as the sampling point. It is also possible to observe that the samples with $0.8 \%$ and $0.2 \%$ volume fraction, have a greater electric resistance for sample points in ranges of 0.6 to 0.8 and $R_{-} E X T=2.2 \mathrm{k} \Omega$. based on this result, the configuration of 0.7 was chosen as a percentage of the positive cycle of the input signal and $2.2 \mathrm{k} \Omega$ as $R_{-} E X T$.

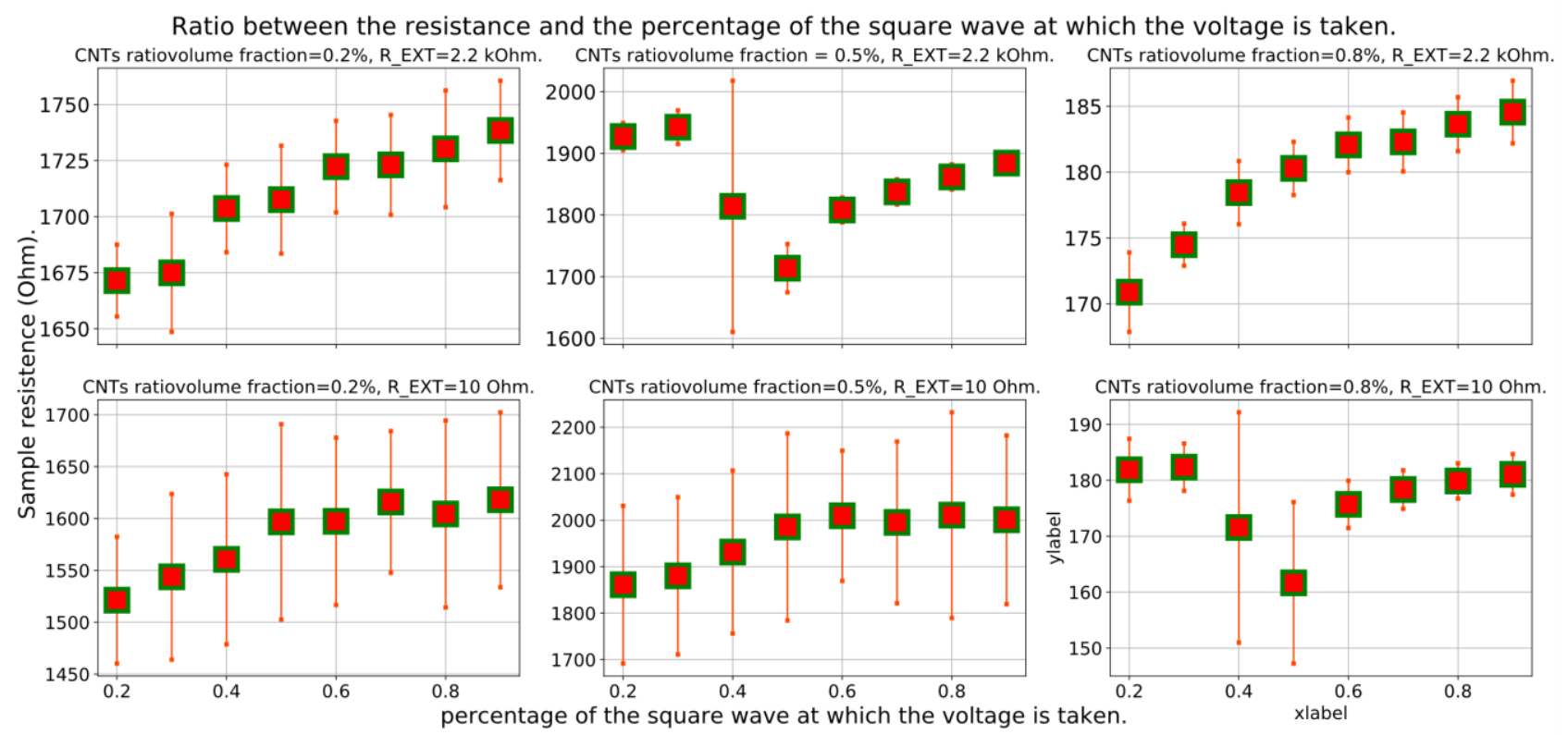

Fig. 5: Ratio between the resistance and the percentage of the square wave at which the voltage is taken.

CNTs volumetric fraction influence can be seen in Fig. 5. When electrical resistance at $0.2 \%$ and $0.5 \%$ volumetric fractions is compared, it is noted that statistically they are equal, however, an abrupt change of almost an order of magnitude occurs when going to $0.8 \%$ of volumetric fraction. this change is related to the percolation threshold, which is the percentage of volume fraction of CNTs necessary for a dielectric matrix to start driving electricity [13].

\subsection{Piezo-resistivity measurements}

The FBGs resolution used in this experimental set up is +/- 1 microstrain, while the DAQ resolution is $0.01 \mathrm{~V}$ and finally, the tolerance of the resistances used to calculate the current along the sample is of $5 \%$ of the value of it.

Fig. 6 shows the electrical responses of the concrete samples with CNTs inclusion, under application of an increasing compression load with a frequency of $1 \mathrm{~Hz}$. It is easy to identify the piezoresistive behavior of the sample with $0.2 \%$ volumetric fraction as almost null, for the simple reason that having so little CNTs, no matter how much a load within the elastic regime is applied, the CNTs will not be in contact [13]. Therefore, the only electricity conduction phenomenon associated with the external load applied on the volume of the sample will due to electronic jump [16]. The gauge factor of the $0.2 \%$ CNTs volume fraction is $\lambda=263.2950$, this means that the resistance of the composite material varies at a rate of $263.2950 \Omega$ per applied strain. This result is inconclusive, because the Fig. 6 shows a large standard deviation, so the final resistance and the initial resistance are statistically equal. 

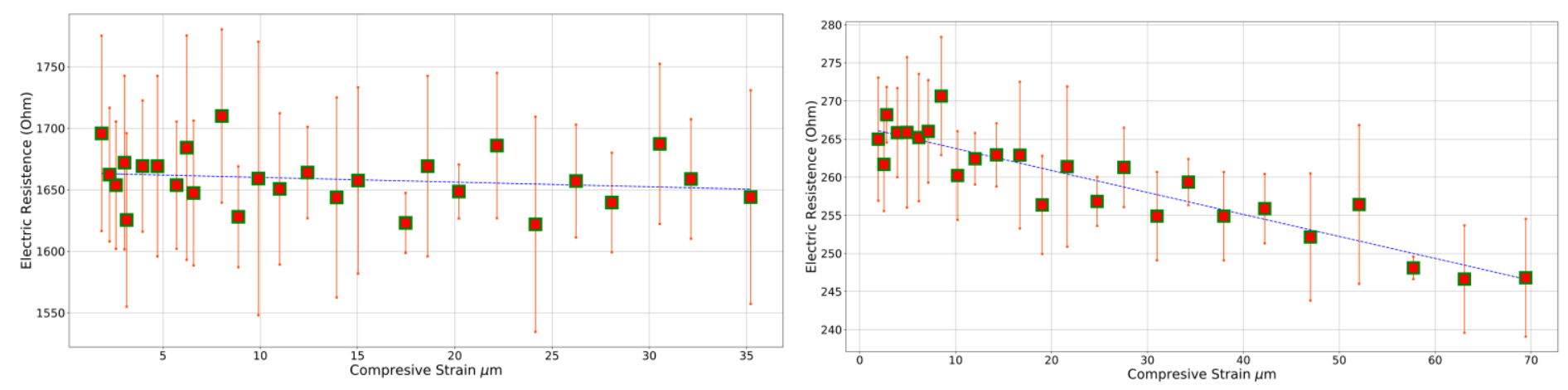

Fig. 6: Strain sensitivity for the $0.2 \%$ (left) and $0.8 \%$ (right) volume fraction sample.

On the other hand, Fig. 6 for $0.8 \%$ CNTs volume fraction demonstrates a well-defined piezoresistive behavior, where the change in electrical resistance varies directly with the strain loads. The gauge factor of the $0.8 \%$ CNTs volume fraction is $\lambda=1308.1694$. It should be noted that the gauge factor increase when percolation threshold is obtained, due to the increasing interparticle distance and with volume expansion when external load is applied [13]. This result is statistically valid, because the error-bars associated with the standard deviation of the initial and final resistance do not cross.

\section{Conclusions}

In this work, a campaign of experimental tests is presented as preliminary work of a study aimed to develop Concrete/CNT composites with self-sensing capabilities for SHM in civil structures.

Tests aimed at studying rheologic and electric properties and strain-sensing capability of cement-based composites doped with carbon nanotubes.

It was observed during the DOE development, that properties such as workability and maximum stress supported to compression by the sample, are inversely proportional to the surfactant and proportions used.

The characterization of the electrical resistance of the compound showed that the selection of the point of the positive cycle of the signal to obtain the voltage and, in turn, the measure of resistance, is a critical parameter, which varies depending on R_EXT and has a similar behavior for the $0.2 \%$ and $0.8 \%$ of CNT load

The origin of the piezoresistive response on the cement matrix composites has been attributed to strain-induced changes in the volume fraction, and it is directly related to the volume fraction of CNTs within the samples and the percolation threshold for Concrete/CNTs composites. Samples with $0.8 \%$ CNTs volume fraction present a higher factor gauge, this is due to the larger proportion of CNTs they contain, greater conductivity is generated by changes in the volumetric fraction induced by strain, this can be corroborated by [13].

As future work, a compound matrix manufactured with a volume fraction of CNTs of $0.8 \%$ within a real engineering component it is proposed, with the objective of performing mechanical tests of the component in healthy initial conditions and then inducing different types of artificial damages in order to acquire data of the resistive behavior of the embedded sensor related to the states. As a final step, implement machine learning classification algorithms is proposed, in order to be trained with the data of the acquired electrical resistance of the sensor to determine when a civil structure is operationally healthy or preventive maintenance is required.

The piezoresistivity measurements accuracy are supported by a high resolution of the equipment used for the data acquisition, thus guaranteeing a minimum dispersion of the results when several repetitions were made.

\section{References}

[1] T. Bock, C. Georgoulas, and T. Linner, "Advanced Construction and Building Technology for Society," 2014.

[2] W. J. W. \& S. J. Holnicki-Szulc, Ed., Smart Technologies for Smart Nations. 2008.

[3] E. T. Thostenson and T. W. Chou, "Carbon nanotube-based health monitoring of mechanically fastened composite joints," Compos. Sci. Technol., vol. 68, no. 12, pp. 2557-2561, 2008.

[4] C. Boller, F. Chang, and Y. Fujino, Encyclopedia of Structural Health Monitoring. 2009. 
[5] Austin R J Downey, Antonella D’Alessandro, Micah Baquera, Enrique Garćia-Maćras, Daniel Rolfes, Filippo Ubertini, Simon Laflamme, Rafael Castro-Triguero, "Damage detection, localization and quantification in conductive smart concrete structures using a resistor mesh model," Eng. Struct., vol. 148, pp. 924-935, 2017.

[6] L. Yu and Z. Tian, Structural Health Monitoring (SHM) in Aerospace Structures. 2016.

[7] C. Boller, F. Chang, and Y. Fujino, [BOOK] Encyclopedia of Structural Health Monitoring. 2009.

[8] S. K. Arsava, J. W. Chong, and Y. Kim, "A novel health monitoring scheme for smart structures," J. Vib. Control, no. October, pp. 1-19, 2014.

[9] S. Lee and S. Park, "Composites: Part A Enhanced dispersion and material properties of multi-walled carbon nanotube composites through turbulent Taylor-Couette flow," Compos. Part A, vol. 95, pp. 118-124, 2017.

[10] B. P. GRADY, CARBON NANOTUBE-POLYMER COMPOSITES, Manufacture, Properties, and Applications. 2011.

[11] M. S. Konsta-Gdoutos and C. A. Aza, "Self sensing carbon nanotube (CNT) and nanofiber (CNF) cementitious composites for real time damage assessment in smart structures," Cem. Concr. Compos., vol. 53, pp. 162-169, 2014.

[12] F. Ubertini, A. L. Materazzi, A. D'Alessandro, and S. Laflamme, "Natural frequencies identification of a reinforced concrete beam using carbon nanotube cement-based sensors," Eng. Struct., vol. 60, pp. 265-275, 2014.

[13] E. Garcia-Macias, A. D’Alessandro, R. Castro-Triguero, D. Perez-Mira, and F. Ubertini, "Micromechanics modeling of the uniaxial strain-sensing property of carbon nanotube cement-matrix composites for SHM applications," Compos. Struct., vol. 163, pp. 195-215, 2017.

[14] E. Garcia-Macias, A. D’Alessandro, R. Castro-Triguero, D. Perez-Mira, and F. Ubertini, "Micromechanics modeling of the electrical conductivity of carbon nanotube cement-matrix composites," Compos. Part B-Engineering, vol. 108, pp. 451-469, 2017.

[15] Hamid Souri, Jaesang Yu, Haemin Jeon, Jae Woo Kim, Cheol-Min Yang,Nam-Ho You, B.J. Yang, "A theoretical study on the piezoresistive response of carbon nanotubes embedded in polymer nanocomposites in an elastic region," Carbon N. Y., vol. 120, pp. 427-437, 2017.

[16] Pham, G. T., "Characterization And Modeling Of Piezo-Resistive Properties Of Carbon Nanotube-Based Conductive Polymer Composites," Florida State University, 2008.

[17] L. Coppola, A. Buoso, and F. Corazza, "Electrical Properties of Carbon Nanotubes Cement Composites for Monitoring Stress Conditions in Concrete Structures," in Performance, Protection and Strengthening of Structures under Extreme Loading, vol. 82, E. Cadoni and M. DiPrisco, Eds. 2011, pp. 118.

[18] M. S. Konsta-Gdoutos and C. A. Aza, "Self sensing carbon nanotube (CNT) and nanofiber (CNF) cementitious composites for real time damage assessment in smart structures," Cem. Concr. Compos., vol. 53, pp. 162-169, 2014.

[19] F. Torabian Isfahani, W. Li, and E. Redaelli, "Dispersion of multi-walled carbon nanotubes and its effects on the properties of cement composites," Cem. Concr. Compos., vol. 74, pp. 154-163, Nov. 2016.

[20] H. Y. Shao, B. M. Chen, B. Li, S. W. Tang, and Z. J. Li, "Influence of dispersants on the properties of CNTs reinforced cement-based materials," Constr. Build. Mater., vol. 131, pp. 186-194, 2017.

[21] G. M. Kim, B. J. Yang, G. U. Ryu, and H. K. Lee, "The electrically conductive carbon nanotube (CNT)/cement composites for accelerated curing and thermal cracking reduction," Compos. Struct., vol. 158, pp. 20-29, 2016.

[22] V. Malagavelli and N. Rao Paturu, "Strength and Workability Characteristics of Concrete by Using Different Super Plasticizers," Int. J. Mater. Eng., vol. 2, no. 1, pp. 7-11, Feb. 2012.

[23] M. S. Konsta-gdoutos, Z. S. Metaxa, and S. P. Shah, "Cement and Concrete Research Highly dispersed carbon nanotube reinforced cement based materials," Cem. Concr. Res., vol. 40, no. 7, pp. 1052-1059, 2010.

[24] E. García-Macías, A. Downey, A. D’Alessandro, R. Castro-Triguero, S. Laflamme, and F. Ubertini, "Enhanced lumped circuit model for smart nanocomposite cement-based sensors under dynamic compressive loading conditions," Sensors Actuators A Phys., vol. 260, pp. 45-57, 2017.

[25] S. Alsadey, "Influence of Superplasticizer on Strength of Concrete," vol. 1, no. 3, pp. 164-166, 2012. 